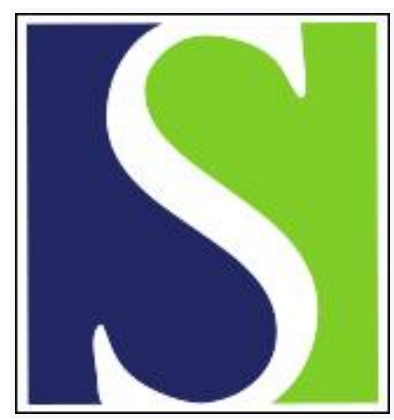

Scand J Work Environ Health 2018;44(4):394-402

https://doi.org/10.5271/sjweh.3730

Published online: 11 Apr 2018, Issue date: 01 Jul 2018

Association of changes in work shifts and shift intensity with change in fatigue and disturbed sleep: a within-subject study

by Härmä M, Karhula K, Ropponen A, Puttonen S, Koskinen A, Ojajärvi A, Hakola T, Pentti J, Oksanen T, Vahtera J, Kivimäki M

The focus of this longitudinal cohort study was to find evidence-based recommendations for shift workers with acute onset of fatigue or disturbed sleep. The results suggest that decreases in the number of night shifts, quick returns, and a preference for quickly forward-rotating shift arrangements are likely to reduce fatigue. Improvements in shift characteristics would be particularly beneficial for employers older than 50 years.

Affiliation: Finnish Institute of Occupational Health, P.O Box 40, FI-00032 TYÖTERVEYSLAITOS. Mikko.Harma@ttl.fi

Refers to the following texts of the Journal: 2006;32(6):413-419

2008;34(3):198-205 2010;36(2):121-133 2011;37(3):173-185

2014;40(6):543-556 2015;41(3):268-279 2017;43(3):191-195

2017;43(5):393-395

The following articles refer to this text: 2020;46(3):293-301;

2020;46(6):557-569; 2021;47(3):181-190; 2021;47(6):446-455;

2023;49(2):108-116

Key terms: disturbed sleep; fatigue; forward-rotating shift; insomnia; night shift; night work; older worker; shift; shift intensity; shift interval; shift work; shift worker; sleep; sleep duration; sleep length; sleep problem; sleepiness; within-subject study; working hours

This article in PubMed: www.ncbi.nlm.nih.gov/pubmed/29641837

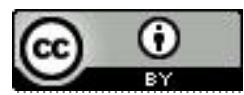




\title{
Association of changes in work shifts and shift intensity with change in fatigue and disturbed sleep: a within-subject study
}

\author{
by Mikko Härmä, MD, PhD, ${ }^{1}$ Kati Karhula, PhD, ${ }^{1}$ Annina Ropponen, PhD, ${ }^{1}$ Sampsa Puttonen, PhD, ${ }^{1}$ Aki Koskinen, BA, ${ }^{1}$ \\ Anneli Ojajärvi, PhD, ${ }^{1}$ Tarja Hakola, MSc, ${ }^{1}$ Jaana Pentti, BSc, ${ }^{3}$ Tuula Oksanen, MD, PhD, ${ }^{1}$ Jussi Vahtera, MD, PhD, ${ }^{2}$ \\ Mika Kivimäki, $P h D^{1,3}$
}

\begin{abstract}
Härmä M, Karhula K, Ropponen A, Puttonen S, Koskinen A, Ojajärvi A, Hakola T, Oksanen T, Vahtera J, Kivimäki M. Association of changes in work shifts and shift intensity with change in fatigue and disturbed sleep: a within-subject study. Scand J Work Environ Health. 2018;44(4):394-402. doi:10.5271/sjweh.3730
\end{abstract}

Objectives The aim of this study was to examine whether changes in work shifts and shift intensity are related to changes in difficulties to fall asleep, fatigue, and sleep length.

\begin{abstract}
Methods Questionnaire responses of hospital employees ( $N=7727,93 \%$ women) in 2008, 2012, 2014 and 2015 were linked to daily-based records of working hours during three months preceding each survey. We used conditional logistic regression and longitudinal fixed-effects analyses to investigate odds ratios (OR) and $95 \%$ confidence intervals (CI) for each $25 \%$ within-individual change in the proportion of working hour characteristics in relation to changes in fatigue, difficulties to fall asleep, and 24-hour sleep length.
\end{abstract}

Results Change in night but not in morning or evening shifts was associated with parallel changes in odds for longer sleep length (OR 1.45, 95\% CI 1.28-1.64) and fatigue during free days (OR 1.38, 95\% CI 1.16-1.64). Similarly, short shift intervals and having $>2$ but not $>4$ consecutive night shifts were associated with increased odds of fatigue during work and difficulties to fall asleep (OR 1.42, 95\% CI 1.19-1.72 and OR 1.10, 95\% CI $1.05-1.19$, respectively). Among workers aged $\geq 50$ years, the associations were the strongest between night shifts and longer sleep (OR 2.24, 95\% CI 1.52-3.81) and between higher proportion of short shift intervals and fatigue during free days (OR 1.68, 95\% CI 1.10-2.54).

Conclusions Among shift workers with fatigue or sleep problems, decreasing the proportion of night shifts and quick returns and giving preference to quickly forward-rotating shift systems may reduce fatigue.

Key terms forward-rotating shift; insomia; older worker; night shift; sleep duration; sleep length; sleep problem; sleepiness; shift interval; shift work; shift worker; working hours.

In Europe, approximately one fifth of the working population is working in shifts (1). Shift work is associated with disturbed sleep and fatigue (2), occupational accidents (3), and several chronic conditions such as cardiovascular disease and type 2 diabetes $(4,5)$. Especially night shifts have been linked to disturbed sleep and fatigue in both cross-sectional and longitudinal studies $(3,6-9)$.

Evidence also suggests that shift work may increase the risk of acute sleep loss (4). Field studies of employees with rotating shift work show that early morning and night shifts in particular induce increased sleepiness and fatigue. Main sleep before early morning shifts is often shortened, as well as main sleep after night shifts (10, 11). Specific shift characteristics, such as quick returns or late evening shifts, may also be associated with disturbed sleep $(12,13)$.

The majority of studies on shift work and sleep are cross-sectional, indicating associations between shift work and sleep although this should be interpreted with caution due to potential selection bias. Even systematic reviews on the interventions and cohort studies that examine the preference of various working hour characteristics in shift work hesitate to provide recommendations for optimal shift work patterns $(2,6,8,14)$. Accordingly, Driscoll et al (6) concluded that there is

\footnotetext{
${ }^{1}$ Finnish Institute of Occupational Health, Finland.

2 University of Turku, Finland.

3 Clinicum, Faculty of Medicine, University of Helsinki, Finland.
} 
insufficient evidence to support any specific shift work patterns, whereas most reviews agree that there is some support for the use of forward-rotating shifts. Forward rotating allows sufficient time between the shifts for recovery and can thus avoid "quick returns" (ie, 11 hours of less free time between the shifts). The literature on the preference of the speed of optimal shift rotation is also controversial. Some previous studies support the use of quickly rotating shift systems (1-2 consecutive night shifts) to improve sleep (15-17) and neurobehavioral performance (18), but contradictory results also exist (19).

Intervention studies on shift work patterns have various limitations. Most intervention studies targeted several shift features simultaneously while comparative data on the relevance of different shift characteristics studied separately is lacking. Studies have focused on regular shift work patterns instead of irregular shift systems, the latter of which are common in service sector. Finally, studies have also relied on subjective information on exposure (working patterns) making them sensitive to memory bias.

To address some of the existing gaps in evidence, we used fixed-effects time-dependent logistic modelling to investigate whether intra-individual changes in the proportion of different working hour characteristics are associated with changes in fatigue and sleep over time. We linked study participants to electronic records of working hours and measured sleep and fatigue repeatedly. Our aim was to find observational evidence for the effects of various shift features needed for evidencebased individual recommendations to prevent disturbed sleep and fatigue among shift workers. We hypothesized that older employees and women are more sensitive to the adverse effects of shift work patterns than younger employees and men, and therefore tested age and gender interactions in the associations of working hour characteristics with changes in fatigue and sleep.

\section{Methods}

\section{Study sample}

In the Finnish Public Sector (FPS) study, survey data of hospital workers responding to $\geq 2$ questionnaires in 2008, 2012, 2014 or 2015 ( $\mathrm{N}=11$ 949) were linked to the payroll data of daily work shifts from three months prior to answering each survey. The response rates to the questionnaires in 2008, 2012, 2014 and 2015 were $72 \%, 71 \%, 67 \%$ and $69 \%$, respectively. Physicians were excluded due to missing information on on-call work. The mean age of the employees was 40.8 years, and 93\% were women (table 1) with various job titles (20). Since our aim was to study whether changes in the continuous working hour characteristics were associated with changes in fatigue and sleep outcomes, we only focused on employees with changes in fatigue and sleep using each person as his or her own control. The longitudinal fixed-effects model used automatically excludes all subjects without a change in the outcome. The final number of participants in the analysis was from 1716 to 2781 (see table 2). The Ethics Committee of the Hospital District of Helsinki and Uusimaa (HUS) approved the FPS study (HUS 1210/2016).

\section{Exposure: shift work patterns}

Payroll-based realized rosters were retrieved from the shift scheduling program (Titania ${ }^{\circledR}$, CGI Finland Ltd, Helsinki, Finland), as described earlier (21). The starting and ending times of the daily working hours were used to define work shifts. Night shifts were defined as $\geq 3$ hours between 23:00-06:00 hours; evening shifts as any time between 18:00-23:00 and not categorized to night shifts; morning shifts as work starting at or after 03:00 hours and ending no later than 18:00 hours $(20,22)$.

Based on the defined work shifts, we studied shift work patterns relating to two main dimensions of the working hours characteristics: the proportion of different work shifts (time of the day) and shift intensity that could be assumed to be associated with sleep (21). For the proportion of work shifts, we calculated the percent

Table 1. Age, gender and the outcome variables in the first survey they joined $(2008,2012$ or $2014, \mathrm{~N}=7727)$. [SD=standard deviation.]

\begin{tabular}{|c|c|c|c|}
\hline & $\begin{array}{l}\text { Participants } \\
\text { (N) }\end{array}$ & Mean (SD) & $\%$ \\
\hline Age (years) & \multicolumn{3}{|c|}{$40.8(10.7)$} \\
\hline$\leq 39$ & 3272 & & 42.4 \\
\hline $40-49$ & 2464 & & 31.9 \\
\hline$\geq 50$ & 1991 & & 25.8 \\
\hline \multicolumn{4}{|l|}{ Gender } \\
\hline Men & 584 & & 7.6 \\
\hline Women & 7143 & & 92.4 \\
\hline \multicolumn{4}{|c|}{ Difficulties to fall asleep } \\
\hline 0-3 times/month & 5103 & & 66.0 \\
\hline 1 time/week & 947 & & 12.3 \\
\hline$\geq 2$ times/week & 927 & & 12.0 \\
\hline Missing & 750 & & 9.7 \\
\hline \multicolumn{4}{|l|}{ Sleep length (hours) } \\
\hline$\leq 6.5$ & 1682 & & 21.8 \\
\hline $7.0-7.5$ & 3212 & & 41.6 \\
\hline$\geq 8.0$ & 2100 & & 27.2 \\
\hline Missing & 733 & & 9.5 \\
\hline \multicolumn{4}{|l|}{ Fatigue during work } \\
\hline 0-3 times/month & 3303 & & 42.8 \\
\hline 1 time/week & 1632 & & 21.1 \\
\hline$\geq 2$ times/week & 2050 & & 26.5 \\
\hline Missing & 742 & & 9.6 \\
\hline \multicolumn{4}{|c|}{ Fatigue during free days } \\
\hline 0-3 times/month & 4701 & & 60.8 \\
\hline 1 time/week & 1139 & & 14.7 \\
\hline$\geq 2$ times/week & 1096 & & 14.2 \\
\hline Missing & 791 & & 10.2 \\
\hline
\end{tabular}


of (i) morning shifts of all work shifts, (ii) evening shifts of all work shifts, (iii) night shifts of all work shifts and (iv) non-day (evening or night) shifts of all work shifts. The working hour characteristics for shift intensity were the percent of (i) long spells (>7) of consecutive work shifts of all consecutive work shifts, (ii) long spells ( $>2$ and $>4$ separately) of consecutive night shifts of all consecutive night shift spells, and (iii) short shift intervals ( $<11$ hours) of all shift intervals. The formulation and basis for these characteristics has been described in more detail before (21). Table 3 shows the mean values and standard deviations of the shift work characteristics used from 2008 to 2015 .

\section{Outcomes and moderators}

Information on sleep and fatigue was self-reported in the surveys. Difficulties to fall asleep, fatigue/tiredness during work shifts and fatigue/tiredness during free days during the last four weeks were asked according to Jenkins $(20,23,24)$. The following options for responses were used: $1=$ "not at all", $2=" 1-3$ days a month", $3=$ "about during one day in a week", $4=$ ="during
2-4 days a week", 5="during 5-6 days a week, $6=$ "every day". We enquired about 24-hour sleep length with half an hour interval with the question "How many hours do you normally sleep during 24 hours?". See table 1 for the distribution of the fatigue/sleep outcomes. Age and gender was obtained from the questionnaire for the moderator analysis.

\section{Statistical methods}

We used longitudinal fixed-effects time-dependent logistic modelling to investigate whether the intra-individual longitudinal changes in working hour characteristics explained the observed changes in the difficulties to fall asleep, fatigue or sleep length between 2008, 2012, 2014 and 2015. The fixed-effects model links changes in working hour characteristics to changes in sleep and fatigue (for both directions) within individuals, thereby controlling for all measured and unmeasured stable individual characteristics and other potential confounders that do not vary over time $(25,26)$. Since our aim was to study whether the changes in the continuous shift work patterns explained changes in fatigue and sleep,

Table 2. The analysed changes and the direction of the changes in the outcomes 2008-2015. Number of participants responding to $\geq 2$ questionnaires ( $\mathrm{N}=11949)$.

\begin{tabular}{|c|c|c|c|c|c|c|c|c|}
\hline & \multicolumn{8}{|c|}{ Surveys 2008, 2012, 2014, 2015} \\
\hline & \multicolumn{2}{|c|}{ Fatigue during work ${ }^{a}$} & \multicolumn{2}{|c|}{ Fatigue during free days ${ }^{a}$} & \multicolumn{2}{|c|}{ Difficulties to fall asleep ${ }^{a}$} & \multicolumn{2}{|c|}{ Sleep length ${ }^{\mathrm{b}}$} \\
\hline & $\mathrm{N}$ & $\%$ & $\mathrm{~N}$ & $\%$ & $\mathrm{~N}$ & $\%$ & $\mathrm{~N}$ & $\%$ \\
\hline Stable low/shorter & 4285 & 35.9 & 4795 & 40.1 & 6580 & 55.1 & 1524 & 12.8 \\
\hline Stable medium & 836 & 7.0 & 957 & 8.0 & 339 & 2.8 & 3092 & 25.6 \\
\hline Stable high/longer & 2557 & 21.4 & 1315 & 11.0 & 903 & 7.6 & 1695 & 14.2 \\
\hline Change between low and high frequency of symptoms & 2301 & 19.3 & 1999 & 16.7 & 1908 & 16.0 & & \\
\hline Increase total & 1349 & 11.3 & 1206 & 10.1 & 1204 & 10.1 & & \\
\hline Low-high & 1087 & 9.1 & 956 & 8.0 & 881 & 7.4 & & \\
\hline Low-high-low & 251 & 2.1 & 242 & 2.0 & 314 & 2.6 & & \\
\hline Low-high-low-high & 11 & 0.1 & 8 & 0.1 & 9 & 0.1 & & \\
\hline Decrease total & 952 & 8.0 & 784 & 6.6 & 704 & 5.9 & & \\
\hline High-low & 806 & 6.7 & 693 & 5.8 & 601 & 5.0 & & \\
\hline High-low-high & 128 & 1.1 & 85 & 0.7 & 93 & 0.8 & & \\
\hline High-low-high-low & 18 & 0.2 & 6 & 0.1 & 10 & 0.1 & & \\
\hline Change between shorter and medium sleep length & & & & & & & 2930 & 24.5 \\
\hline Increase total & & & & & & & 1168 & 9.8 \\
\hline Shorter-medium & & & & & & & 831 & 7.0 \\
\hline Shorter-medium-shorter & & & & & & & 290 & 2.4 \\
\hline Shorter-medium-shorter-medium & & & & & & & 47 & 0.4 \\
\hline Decrease total & & & & & & & 1762 & 14.7 \\
\hline Medium-shorter & & & & & & & 1471 & 12.3 \\
\hline Medium-shorter-medium & & & & & & & 263 & 2.2 \\
\hline Medium-shorter-medium-shorter & & & & & & & 28 & 0.2 \\
\hline Change between medium and longer sleep length & & & & & & & 3068 & 25.7 \\
\hline Increase total & & & & & & & 1305 & 10.9 \\
\hline Medium-longer & & & & & & & 948 & 7.9 \\
\hline Medium-longer-medium & & & & & & & 329 & 2.8 \\
\hline Medium-longer-medium-longer & & & & & & & 28 & 0.2 \\
\hline Decrease total & & & & & & & 1763 & 14.8 \\
\hline Longer-medium & & & & & & & 1424 & 11.9 \\
\hline Longer-medium-longer & & & & & & & 280 & 2.3 \\
\hline Longer-medium-Ionger-medium & & & & & & & 59 & 0.5 \\
\hline
\end{tabular}

${ }^{a}$ Low: 0-3 times/month, medium: 1 time/week, high: $\geq 2$ times/week.

${ }^{\text {b }}$ Shorter: $\leq 6.5$ hours/day, medium: 7.0-7.5 hours/day, longer: $\geq 8$ hours/day 
Table 3. The average proportion (\%) of working hour characteristics during the past 91 days in 2008-2015. ( $N=7727)$. [SD=standard deviation.]

\begin{tabular}{|c|c|c|c|c|c|c|c|c|}
\hline & \multicolumn{2}{|c|}{2008} & \multicolumn{2}{|c|}{2012} & \multicolumn{2}{|c|}{2014} & \multicolumn{2}{|c|}{2015} \\
\hline & Mean & SD & Mean & SD & Mean & SD & Mean & SD \\
\hline \multicolumn{9}{|l|}{ Work shifts } \\
\hline Morning/all shifts & 64.9 & 28.3 & 64.8 & 28.2 & 65.0 & 28.9 & 65.6 & 29.8 \\
\hline Evening/all shifts & 19.1 & 18.5 & 19.3 & 18.9 & 19.5 & 19.5 & 19.3 & 19.9 \\
\hline Night/all shifts & 9.8 & 17.7 & 9.5 & 18.1 & 9.6 & 18.2 & 9.7 & 18.2 \\
\hline Non-day/all shifts & 29.1 & 28.0 & 29.2 & 28.0 & 29.2 & 28.8 & 29.1 & 29.4 \\
\hline \multicolumn{9}{|l|}{ Shift intensity } \\
\hline Long spells of work shifts/all spells of work shifts & 2.4 & 6.4 & 2.3 & 6.6 & 2.2 & 6.4 & 2.2 & 6.3 \\
\hline$>2$ consecutive night shifts/all spells of consecutive night shifts & 40.2 & 48.8 & 37.8 & 48.2 & 36.5 & 47.9 & 36.4 & 47.9 \\
\hline$>4$ consecutive night shifts/all spells of consecutive night shifts & 9.6 & 24.9 & 10.1 & 25.2 & 10.2 & 25.1 & 10.3 & 25.4 \\
\hline Short ( $<11$ hours) shift intervals/all shift intervals & 12.3 & 15.3 & 11.1 & 14.0 & 10.8 & 13.9 & 9.8 & 13.2 \\
\hline
\end{tabular}

the fixed-effects model excluded all employees without changes in the outcome between the four questionnaires. Comparable to a case-crossover design, the design used investigated the question, "Did anything unusual or different take place before the onset of the event?" Casecrossover designs examine the transient effects of an intermittent exposure on acute outcomes and apply best if the exposure is intermittent, the effect on risk is immediate and transient, and the outcome is abrupt $(25,27)$. In our design, the association of exposure to outcome was studied during the three-month period prior to each survey, and the wash-out period was from 9-45 months, depending on the time between the separate surveys.

In relation to fatigue and sleep problems, the participants were non-cases in the survey waves where they had symptoms of fatigue or difficulties to fall asleep $\leq 3$ times per month. They were cases in the study waves where they reported the corresponding symptoms to occur $\geq 2$ times per week. Since both shorter and longer sleep duration may be associated with poorer health and higher need for recovery (28), the participants were non-cases (had normal sleep length) in the study waves where they reported sleeping 7.0-7.5 hours during the 24 hours. They were cases in the waves where they reported either shorter $(\leq 6.5$ hours) or longer sleep $(\geq 8$ hours). We calculated odds ratios (OR) and their $95 \%$ confidence intervals $(\mathrm{CI})$ for any $25 \%$ increase in the continuous exposure variables (the variables describing the proportion of different work shifts and shift intensity, see below) in relation to the outcomes (fatigue during work, fatigue during free days, difficulties to fall asleep, short sleep and long sleep). For example, having two rather than one night shifts during a working week of five days results in a $20 \%$ increase in the prevalence of the proportion of night shifts. Based on interactions with $\mathrm{P}<0.10$ (29), a stratified analysis of age $(\leq 39,40-49$ and $\geq 50$ years) and gender was conducted.

The longitudinal fixed-effects model combines all increases and decreases of the repeated data giving only one risk estimate for the association of a change in exposure to the change in outcome. However, we made additional stratified sensitivity analyses to compare the changes in exposure for an increase or decrease of the same outcome to see whether the associations would be reversed. For that purpose, subsamples of different groups with only a single increase or decrease in the outcome (table 2) were selected. Because this leads to decreased power compared to the original analysis, we made the stratified analysis only to few variables showing a significant association with the outcomes in the earlier analysis.

The statistical analyses were conducted with SAS 9.4 (SAS Institute Inc, Cary, NC, USA).

\section{Results}

\section{Description of the outcome and exposure variables}

Of all the employees, $12.0 \%$ had difficulties to fall asleep at least twice a week; $21.8 \%$ slept $\leq 6.5$ hours and $27.2 \% \geq 8.0$ hours; $26.5 \%$ had fatigue during working hours at least twice a week; and $14.2 \%$ suffered fatigue during free days (table 1). The mean values and standard deviations of the working hour characteristics are shown in table 3.

\section{Work shifts}

Night and non-day shifts were significantly associated with more fatigue during free days and longer 24-hour sleep length (tables 4-5). On the other hand, morning shifts were associated with significantly lower odds for fatigue during free days, difficulties to fall asleep and longer sleep length (tables 4-5). Evening shifts were associated with difficulties to fall asleep only (OR 1.38, 95\% CI 1.16-1.60). The association between non-day shifts and longer sleep length was higher among men (OR 1.68, 95\% CI 1.16-2.42) than women (OR 1.19, 95\% CI 1.08-1.31) (test of interaction, $\mathrm{P}<0.091$ ). The association of night shifts with longer sleep length increased with age ( $\leq 39$ years: OR $1.28,95 \%$ CI 1.08 1.49 ; 40-49 years: OR 1.72, 95\% CI 1.28-2.25; and 
Table 4. The association of working hour characteristics with fatigue during work and free days. Longitudinal fixed-effects model. Odds ratio (OR) refer to every $25 \%$ increase in the occurrence of the 3-month prevalence $(\%)$ of the continuous exposure variables. Significant interactions according to age and gender. [Cl=confidence interval.]

\begin{tabular}{|c|c|c|c|c|c|c|c|c|}
\hline & \multicolumn{4}{|c|}{ Fatigue during work } & \multicolumn{4}{|c|}{ Fatigue during free days } \\
\hline & $\mathrm{N}$ & OR $(95 \% \mathrm{Cl})$ & Age & Gender & $\mathrm{N}$ & OR $(95 \% \mathrm{Cl})$ & Age & Gender \\
\hline \multicolumn{9}{|l|}{ Work shifts } \\
\hline Morning shifts & 2059 & $0.98(0.88-1.08)$ & - & - & 1753 & $0.86(0.78-0.95)$ & - & - \\
\hline Evening shifts & 2059 & $1.13(0.98-1.28)$ & - & - & 1753 & $1.10(0.93-1.28)$ & - & 0.092 \\
\hline Night shifts & 2059 & $1.05(0.90-1.22)$ & - & - & 1753 & $1.38(1.16-1.64)$ & - & 0.094 \\
\hline Non-day shifts & 2059 & $1.10(0.98-1.22)$ & - & - & 1753 & $1.25(1.10-1.42)$ & - & - \\
\hline \multicolumn{9}{|l|}{ Shift intensity } \\
\hline Long spells of work shifts & 2059 & $1.10(0.84-1.42)$ & - & - & 1753 & $1.25(0.98-1.64)$ & - & - \\
\hline$>2$ consecutive night shifts & 2059 & $\begin{array}{r}1.10(1.05-1.19) \\
1.13(1.05-1.22)^{a}\end{array}$ & - & - & 1753 & $\begin{array}{r}1.10(1.03-1.16) \\
1.05(0.98-1.13)^{\mathrm{a}}\end{array}$ & - & - \\
\hline$>4$ consecutive night shifts & 2059 & $\begin{array}{r}1.05(0.95-1.13) \\
1.05(0.93-1.16)^{\mathrm{a}}\end{array}$ & - & - & 1753 & $\begin{array}{r}1.00(0.93-1.10) \\
1.03(0.86-1.19)^{\text {a }}\end{array}$ & - & - \\
\hline Short shift intervals & 2081 & $1.42(1.19-1.72)$ & - & - & 1783 & $1.25(1.03-1.49)$ & 0.092 & - \\
\hline
\end{tabular}

${ }^{a}$ Controlled for the $\%$ of night shifts.

Table 5. The association of working hour characteristics with difficulties to fall asleep and 24-hour sleep length. Fixed-effects model. Odds ratio $(\mathrm{OR})$ refer to every $25 \%$ increase in the occurrence of the 3-month prevalence $(\%)$ of the continuous exposure variables. Significant interactions according to age and gender. [Cl=confidence interval.]

\begin{tabular}{|c|c|c|c|c|c|c|c|c|c|c|}
\hline & \multicolumn{3}{|c|}{ Difficulties to fall asleep } & \multicolumn{3}{|c|}{ Short 24-hour sleep } & \multicolumn{4}{|c|}{ Long 24-hour sleep } \\
\hline & $\mathrm{N}$ & OR $(95 \% \mathrm{Cl})$ & Age & $\mathrm{N}$ & OR (95\% Cl) & Gender & $\mathrm{N}$ & OR (95\% Cl) & Age & Gender \\
\hline \multicolumn{11}{|l|}{ Work shifts } \\
\hline Morning & 1725 & $0.80(0.70-0.88)$ & - & 2583 & $1.08(0.98-1.16)$ & - & 2755 & $0.80(0.74-0.88)$ & - & - \\
\hline Evening & 1725 & $1.38(1.16-1.60)$ & - & 2583 & $0.93(0.82-1.08)$ & - & 2755 & $1.00(0.88-1.13)$ & - & - \\
\hline Night & 1725 & $1.03(0.86-1.22)$ & - & 2583 & $0.88(0.76-1.03)$ & - & 2755 & $1.45(1.28-1.64)$ & 0.019 & - \\
\hline Non-day & 1725 & $1.22(1.08-1.38)$ & - & 2583 & $0.90(0.82-1.03)$ & - & 2755 & $1.22(1.10-1.31)$ & - & 0.091 \\
\hline \multicolumn{11}{|l|}{ Shift intensity } \\
\hline Long spells of work shifts & 1725 & $1.03(0.78-1.38)$ & 0.099 & 2583 & $1.13(0.90-1.45)$ & - & 2755 & $0.80(0.63-1.03)$ & - & - \\
\hline$>2$ consecutive night shifts & 1725 & $\begin{array}{r}1.13(1.05-1.22) \\
1.16(1.08-1.25)^{a}\end{array}$ & - & 2583 & $\begin{array}{c}1.00(0.93-1.05) \\
1.00(0.95-1.08)^{a}\end{array}$ & - & 2755 & $\begin{array}{r}1.03(0.98-1.08) \\
0.98(0.93-1.03)^{a}\end{array}$ & - & - \\
\hline$>4$ consecutive night shifts & 1725 & $\begin{array}{r}0.95(0.86-1.05) \\
0.93(0.82-1.03)^{a}\end{array}$ & - & 2583 & $\begin{array}{c}0.93(0.86-1.00) \\
0.95(0.86-1.00)^{a}\end{array}$ & $\begin{array}{l}0.067 \\
0.068\end{array}$ & 2755 & $\begin{array}{r}1.10(1.05-1.19) \\
1.03(0.93-1.10)^{\mathrm{a}}\end{array}$ & - & - \\
\hline Short shift intervals & 1745 & $1.38(1.13-1.64)$ & - & 2615 & $1.00(0.86-1.16)$ & - & 2781 & $0.90(0.78-1.03)$ & - & - \\
\hline
\end{tabular}

${ }^{a}$ Controlled for the $\%$ of night shifts.

$\geq 50$ years: OR 2.24, 95\% CI 1.52-3.81; age interaction, $\mathrm{P}<0.019)$.

\section{Shift intensity}

Short shift intervals were associated with fatigue during work (OR 1.42, 95\% CI 1.19-1.72), fatigue during free days (OR 1.25, 95\% CI 1.03-1.49) and difficulties to fall asleep (OR 1.38, 95\% CI 1.13-1.64). For fatigue during free days, the association with short shift intervals was found only in the youngest and oldest age groups $(\leq 39$ years: OR 1.49, 95\% CI 1.13-1.95; 40-49 years: OR $0.90,95 \%$ CI $0.65-1.28$; and $\geq 50$ years: OR $1.68,95 \%$ CI 1.10-2.54; interaction, $\mathrm{P}<0.092$ ).

The proportion of $>2$ and $>4$ consecutive night shifts were associated with increased fatigue during work and free-days and difficulties to fall asleep (tables 4-5). When controlled for the proportion of night shifts, only the proportion of $>2$ consecutive night shifts was significantly associated with fatigue during work (OR $1.13,95 \%$ CI 1.05-1.22) and difficulties to fall asleep (OR 1.16, 95\% CI 1.08-1.25).

\section{Stratified analysis}

In a stratified sensitivity analysis, we studied the association of work shifts and shift intensity with an increase or decrease of the outcome. A decrease in fatigue during free days was associated with lower odds for higher number of night shifts ( $=700$, OR $0.57,95 \%$ CI 0.40 0.84 ). Similarly, a decrease in fatigue during work was associated with lower odds for short shifts intervals $(\mathrm{N}=1214$, OR $0.54,95 \%$ CI $0.39-0.76)$ and a decrease in long 24-hour sleep was associated with lower odds for over two consecutive night shifts $(\mathrm{N}=1563$, OR 0.82 , 95\% CI 0.76-0.90). An increase in fatigue during free days was associated with higher odds for the number of night shifts ( $\mathrm{N}=1025$, OR $1.16,95 \%$ CI $0.86-1.56)$ and an increase in long 24-hour sleep was associated with higher odds for $>4$ consecutive night shifts $(\mathrm{N}=1140$, OR 1.16, 95\% CI 1.03-1.35). However, an increase of fatigue during work was not associated to increased but to decreased odds for short shift intervals $(\mathrm{N}=1214$, OR $0.54,95 \%$ CI $0.39-0.76$ ). 


\section{Discussion}

The aim of this study was to examine the association of changes in work shifts and shift intensity with changes in fatigue and sleep using longitudinal registry data of working hours and repeated self-reported data on sleep and fatigue. We found that night shifts were associated with increased fatigue during free days and longer sleep length in a within-individual analysis. Short shift intervals and having $>2$ consecutive night shifts were associated with higher fatigue during work and free days, as well as increased difficulties to fall asleep. Evening shifts were associated with increased difficulties to fall asleep. The age interactions showed stronger association among elderly workers ( $\geq 50$-year-old participants) between changes in night shifts and longer 24-hour sleep, as well between short shift intervals on fatigue during free days. Our large cohort study with 7727 hospital employees in shift work appears to be the first to examine specific working hour characteristics using objective repeat data of daily working hours preceding self-reports of fatigue and sleep.

According to our earlier results using a traditional mixed-model logistic regression, long-term exposure to shift work with night shifts was associated with fatigue during free days and longer 24-hour sleep length compared to day work when adjusting for baseline differences and the main confounders (20). Furthermore, shifting from day to shift work was associated with long sleep, while shifting from shift to day work was less likely to be associated with long sleep and fatigue during both work and free days compared to those who did not change their work schedule. The current study uses fixed-effects time-dependent logistic regression and adds to our earlier results by analysing the association of the more specific working hour characteristics with fatigue and sleep among employees with an onset or cessation of their fatigue and sleep problems.

The results indicate that a change in the proportion of night shifts, but not evening shifts, was associated to corresponding increases or decreases in fatigue during free days and longer sleep. In a subsample of the same population using objective sleep recording, shift work was found to be associated with acute shift-dependent truncation of sleep length during only the early morning and night shifts (11). The current results suggest that the observed increase of fatigue during free days and longer 24-hour sleep length are indications for increased need for recovery during free days reflecting the acute effects of shift work on sleep.

In addition to the night shifts, a change in the proportion of quick returns of $<11$ hours between the work shifts was associated with fatigue during work and free days as well as an increase in difficulties to fall asleep. Our results support the earlier findings where quick returns predicted sleep disturbances and fatigue after one-year follow-up compared to a control group (30). Quick returns in hospital work are mostly caused by evening-morning shift combinations (13), and they are associated with increased sleepiness and short sleep duration due to prolonged sleep latency after the evening shifts and more abrupt ending of the main sleep before the morning shift $(11,31)$. It is thus probable that the observed increased risk for difficulties to fall asleep in association with evening shifts in this study was partly due to the quick returns associated with combined evening and morning shifts.

The results also showed that a change in the proportion of $>2$ but not $>4$ consecutive night shifts was associated with an increased fatigue during and after work and more difficulties to fall asleep. The result of increased fatigue following $>2$ consecutive night shifts stayed after controlling for the number of nights shifts during the 3-month recording period. The finding supports the earlier results that showed a decrease of insomnia with a change from 3 to 1-2 consecutive night shifts (15-17). Having $>4$ consecutive night shifts was, however, not associated with fatigue or insomnia. Since the percentage of night shifts was only $10 \%$ in this sample, having $>4$ consecutive night shifts was rare and possibly explained the obtained negative results for their association with sleep. We also did not measure fatigue and sleep during the night shifts but over the whole shift system. The obtained results on the association of the speed of shift rotation with fatigue and sleep cannot be generalized to regular shifts systems outside standard hospital work.

The obtained age interactions showed a clearer association between the proportion of night shifts and longer sleep and between the proportion of short shift intervals and fatigue during free days among ageing employees ( $\geq 50$ years). This is in line with our earlier results showing that after changing from shift work to day work, the decrease of fatigue during free days was most evident among the $\geq 50$-year-old employees (20). However, we could not observe an age interaction with the number of consecutive night shifts and sleep, as indicated earlier by a laboratory (32) and an intervention (16) study. The observed results of the age-related association of working hour characteristics with fatigue during free days and longer sleep length seem to indicate a generally increased need for recovery of the ageing population $(33,34)$.

The strengths of our study are a large and representative sample and the use of objective and precise exposure data of working hour characteristics optimal for the analysis of complicate and changing working time patterns (21). The within-subjects fixed-effects regression uses the participants as their own controls, a strategy controlling for all measured and unmeasured 
individual characteristics and other potential confounders that do not vary over time (26). Since the variability of individual symptoms on fatigue and sleep are highly individual, the use of a within-subjects fixed-effects analysis removing time-invariant confounding is a clear strength of the study. The use of objective exposure information decreases possibilities for reversed causality. The observed results based on the fixed-effects analysis approach are generalizable, however, only to those with changes in sleep and/or fatigue answering thus to the question "did anything unusual in working hours take place before the onset of fatigue/change in sleep length? The method used is comparable to a casecrossover design $(25,27)$.

Although our analysis controls for all time-invariant confounders, time-dependent changes affecting both the shift work patterns and sleep could be a source of bias in our results. Controlling for mental and physical health could, however, lead to over-adjustment since sleep disturbances are also a plausible pathway from shift work to acute and chronic health impairments (4, $35)$. In this population changing from shift to day work is much more common than changing from day to shift work (24). Since hospital employees with emerging physical and mental health problems or chronic insomnia in shift work are more likely to change to less rather than more demanding shift work patterns, any uncontrolled time-variant confounding, including an increase in the number of small children in the family, should dilute the observed associations between the changes in working hour characteristics and changes in fatigue/ sleep. A time-variant increase in the length of working hours could also increase the number of executed work shifts. To control for that we used the percentage instead of absolute values of the working hour characteristics. Sensitivity analyses including a time-variant confounder part-time/full-time status did not also change the results. A limitation of the current study are the specific shift work patterns of most hospitals in Europe with a lower proportion of night shifts and many quick returns (36) meaning that the obtained results cannot be generalized to all shifts systems.

Our focus was on finding evidence-based recommendations for occupational health services dealing with shift workers with acute onset of fatigue or disturbed sleep. The results show that changes in the proportion of night shifts, quick returns, and the number of $>2$ consecutive night shifts are associated with changes in fatigue during work, fatigue during free days, difficulties to fall asleep and longer 24-hour sleep. Stratified sensitivity analysis according to the direction of the change in the outcomes showed that decreases in fatigue and other sleep/wake problems were associated with significant decreases in exposure. The results thus indicate that -among shift workers with fatigue or sleep problems - decreasing the proportion of night shifts and quick returns and preferring quickly forward-rotating shift systems may reduce fatigue. Based on the observed age interactions, improvement of the shift characteristics would be particularly beneficial for employees aged $\geq 50$ years.

\section{Acknowledgement}

This work was supported by a grant from the Finnish Work Environment Fund (no 114317) and NordForsk (74809). MK is supported by NordForsk (75021) and the Finnish Work Environment Fund (no 115421).

\section{References}

1. Eurofound. Working time patterns for sustainable work. Luxembourg: Publications Office of the European Union; 2017.

2. Sallinen M, Kecklund G. Shift work, sleep, and sleepiness - differences between shift schedules and systems. Scand J Work Environ Health 2010 Mar;36(2):121-33. http://dx.doi. org/10.5271/sjweh.2900.

3. Wagstaff AS, Sigstad Lie JA. Shift and night work and long working hours--a systematic review of safety implications. Scand J Work Environ Health 2011 May;37(3):173-85. http://dx.doi.org/10.5271/sjweh.3146.

4. Kecklund G, Axelsson J. Health consequences of shift work and insufficient sleep. BMJ 2016 Nov;355:i5210. http:// dx.doi.org/10.1136/bmj.i5210

5. Vyas MV, Garg AX, Iansavichus AV, Costella J, Donner A, Laugsand LE et al. Shift work and vascular events: systematic review and meta-analysis. BMJ 2012 Jul;345:e4800. http://dx.doi.org/10.1136/bmj.e4800.

6. Driscoll TR, Grunstein RR, Rogers NL. A systematic review of the neurobehavioural and physiological effects of shiftwork systems. Sleep Med Rev 2007 Jun;11(3):179-94. http://dx.doi.org/10.1016/j.smrv.2006.11.001.

7. Linton SJ, Kecklund G, Franklin KA, Leissner LC, Sivertsen $\mathrm{B}$, Lindberg $\mathrm{E}$ et al. The effect of the work environment on future sleep disturbances: a systematic review. Sleep Med Rev 2015 Oct;23:10-9. http://dx.doi.org/10.1016/j. smrv.2014.10.010.

8. Neil-Sztramko SE, Pahwa M, Demers PA, Gotay CC. Health-related interventions among night shift workers: a critical review of the literature. Scand J Work Environ Health 2014 Nov;40(6):543-56. http://dx.doi.org/10.5271/ sjweh.3445.

9. Åkerstedt T, Nordin M, Alfredsson L, Westerholm P, Kecklund G. Sleep and sleepiness: impact of entering or leaving shiftwork--a prospective study. Chronobiol Int 2010 Jul;27(5):987-96. http://dx.doi.org/10.3109/07420528.2010 .489423 . 
10. Rosa RR, Härmä M, Pulli K, Mülder M, Näsman O. Rescheduling a three shift system at a steel rolling mill: effects of a one hour delay of shift starting times on sleep and alertness in younger and older workers. Occup Environ Med 1996 Oct;53(10):677-85. http://dx.doi.org/10.1136/ oem.53.10.677.

11. Karhula K, Härmä M, Sallinen M, Hublin C, Virkkala J, Kivimäki $M$ et al. Job strain, sleep and alertness in shift working health care professionals -- a field study. Ind Health 2013;51(4):406-16. http://dx.doi.org/10.2486/ indhealth.2013-0015.

12. Åkerstedt T. Shift work and disturbed sleep/wakefulness. Occup Med (Lond) 2003 Mar;53(2):89-94. http://dx.doi. org/10.1093/occmed/kqg046.

13. Vedaa Ø, Harris A, Bjorvatn B, Waage S, Sivertsen B, Tucker $\mathrm{P}$ et al. Systematic review of the relationship between quick returns in rotating shift work and health-related outcomes. Ergonomics 2016;59(1):1-14. http://dx.doi.org/10.1080/00 140139.2015.1052020.

14. Bambra CL, Whitehead MM, Sowden AJ, Akers J, Petticrew MP. Shifting schedules: the health effects of reorganizing shift work. Am J Prev Med 2008 May;34(5):427-34. http:// dx.doi.org/10.1016/j.amepre.2007.12.023.

15. Hakola T, Härmä M. Evaluation of a fast forward rotating shift schedule in the steel industry with a special focus on ageing and sleep. J Hum Ergol (Tokyo) 2001 Dec;30(12):315-9.

16. Härmä M, Hakola T, Kandolin T, Sallinen M, Virkkala J, Bonnefond A et al. A controlled intervention study on the effects of a very rapidly forward rotating shift system on sleep-wakefulness and well-being among young and elderly shift workers. Int J Psychophysiol 2006 Jan;59(1):70-9. http://dx.doi.org/10.1016/j.ijpsycho.2005.08.005.

17. Viitasalo K, Kuosma E, Laitinen J, Härmä M. Effects of shift rotation and the flexibility of a shift system on daytime alertness and cardiovascular risk factors. Scand J Work Environ Health 2008 Jun;34(3):198-205. http://dx.doi. org/10.5271/sjweh.1228.

18. Magee M, Sletten TL, Ferguson SA, Grunstein RR, Anderson C, Kennaway DJ et al. Associations between number of consecutive night shifts and impairment of neurobehavioral performance during a subsequent simulated night shift. Scand J Work Environ Health 2016 May;42(3):217-27.

19. Karlson B, Eek F, Orbaek P, Osterberg K. Effects on sleeprelated problems and self-reported health after a change of shift schedule. J Occup Health Psychol 2009 Apr;14(2):97109. http://dx.doi.org/10.1037/a0014116.

20. Härmä M, Karhula K, Puttonen S, Ropponen A, Koskinen A, Ojajärvi A et al. Shift work with and without night work as a risk factor for fatigue and changes in sleep length: A cohort study with linkage to records on daily working hours. J Sleep Res 2018 Jan. [Epub ahead of print]. http://dx.doi. org/10.1111/jsr.12658.

21. Härmä M, Ropponen A, Hakola T, Koskinen A, Vanttola
$\mathrm{P}$, Puttonen $\mathrm{S}$ et al. Developing register-based measures for assessment of working time patterns for epidemiologic studies. Scand J Work Environ Health 2015 May;41(3):268 79. http://dx.doi.org/10.5271/sjweh.3492.

22. Karhula K, Puttonen S, Ropponen A, Koskinen A, Ojajärvi A, Kivimäki $\mathrm{M}$ et al. Objective working hour characteristics and work-life conflict among hospital employees in the Finnish public sector study. Chronobiol Int 2017;34(7):87685. http://dx.doi.org/10.1080/07420528.2017.1329206.

23. Jenkins CD, Stanton BA, Niemcryk SJ, Rose RM. A scale for the estimation of sleep problems in clinical research. J Clin Epidemiol 1988;41(4):313-21. http://dx.doi. org/10.1016/0895-4356(88)90138-2.

24. Härmä M, Koskinen A, Ropponen A, Puttonen S, Karhula $\mathrm{K}$, Vahtera J et al. Validity of self-reported exposure to shift work. Occup Environ Med 2017 Mar;74(3):228-30. http:// dx.doi.org/10.1136/oemed-2016-103902.

25. Maclure M, Mittleman MA. Should we use a case-crossover design? Ann Rev Public Health 2000;21:193-221. http:// dx.doi.org/10.1146/annurev.publhealth.21.1.193.

26. Burdorf A, LaMontagne AD. Analytical strategies to determine whether job strain is an important risk factor for occurrence of low-back pain. Scand J Work Environ Health 2017 Sep;43(5):393-5. http://dx.doi.org/10.5271/ sjweh.3657.

27. Lombardi DA. Advances in occupational traumatic injury research. Scand J Work Environ Health 2017 May;43(3):191-5. http://dx.doi.org/10.5271/sjweh.3640.

28. Kronholm E, Härmä M, Hublin C, Aro AR, Partonen T. Self-reported sleep duration in Finnish general population. J Sleep Res 2006 Sep;15(3):276-90. http://dx.doi. org/10.1111/j.1365-2869.2006.00543.x.

29. Greenland S, Rothman KJ. Concepts of interaction. Modern Epidemiology. USA, New York: Lippincott-Raven; 1998. p. 329-42.

30. Flo E, Pallesen S, Moen BE, Waage S, Bjorvatn B. Short rest periods between work shifts predict sleep and health problems in nurses at 1-year follow-up. Occup Environ Med 2014 Aug;71(8):555-61. http://dx.doi.org/10.1136/ oemed-2013-102007.

31. Vedaa Ø, Mørland E, Larsen M, Harris A, Erevik E, Sivertsen B et al. Sleep detriments associated with quick returns in rotating shift work: A diary study. J Occup Environ Med 2017 Jun;59(6):522-7. http://dx.doi. org/10.1097/JOM.0000000000001006.

32. Härmä MI, Hakola T, Åkerstedt T, Laitinen JT. Age and adjustment to night work. Occup Environ Med 1994 Aug;51(8):568-73. http://dx.doi.org/10.1136/oem.51.8.568.

33. Gommans FG, Jansen NW, Stynen D, de Grip A, Kant I. Need for recovery across work careers: the impact of work, health and personal characteristics. Int Arch Occup Environ Health 2015 Apr;88(3):281-95. http://dx.doi.org/10.1007/ s00420-014-0956-3.

34. Jansen N, Kant I, van Amelsvoort L, Nijhuis F, van den Brandt P. Need for recovery from work: evaluating short- 
term effects of working hours, patterns and schedules. Ergonomics 2003 Jun;46(7):664-80. http://dx.doi.org/10.1 080/0014013031000085662.

35. Härmä M, Kompier MA, Vahtera J. Work-related stress and health--risks, mechanisms and countermeasures. Scand J Work Environ Health 2006 Dec;32(6):413-9. http://dx.doi. org/10.5271/sjweh.1047.
36. Griffiths P, Dall'Ora C, Simon M, Ball J, Lindqvist R, Rafferty AM et al.; RN4CAST Consortium. Nurses' shift length and overtime working in 12 European countries: the association with perceived quality of care and patient safety. Med Care 2014 Nov;52(11):975-81. http://dx.doi. org/10.1097/MLR.0000000000000233.

Received for publication: 18 January 2018 